\title{
REDES DE CIUDADES Y CAMBIO CLIMÁTICO: LA COLABORACIÓn DE CIUDAD DE MÉXICO CON ICLEI Y C40
}

\section{City netuorks and Climate Change: Mexico City's Collaboration with ICLEl and C40}

\author{
María Luisa Azpíroz \\ Universidad Panamericana, Escuela de Comunicación \\ E-mail:mazpiroz@up.edu.mx
}

0 Autor

Las grandes ciudades están cobrando un papel cada vez más relevante como actores políticos a nivel nacional e internacional. En desarrollo sostenible y cambio climático han demostrado su capacidad de llevar la iniciativa incluso por delante de los Gobiernos nacionales, implementando proyectos, adoptando compromisos a nivel internacional, colaborando y compartiendo conocimientos con otras ciudades mediante redes. A este respecto, el presente artículo examina la colaboración de Ciudad de México con las redes de ciudades ICLEI y C40. Para ello se analizó información oficial y se realizaron entrevistas a trabajadores de la Secretaría de Medioambiente de Ciudad de México, ICLEI y C40.

Big cities are gaining an increasing relevant role as political actors at the national and international level. In the areas of sustainable development and climate change, cities have demonstrated their ability to take the initiative even ahead of national governments, implementing projects, adopting international commitments, collaborating and sharing knowledge through networks with other cities. In this regard, this article examines the collaboration of Mexico City with the city networks ICLEI and C40. To this end, official information was analyzed, and interviews were held with workers from the Secretariat of the Environment of Mexico City, ICLEI and C40.

Redes de ciudades; cambio climático; Ciudad de México; ICLEI; C40.

City Networks; Climate Change; Mexico City; ICLEI; C40.

Key uords 


\section{Introducción. Las ciudades como actores internacionales contra el cambio climático}

Hoy día, los Estados ya no son los únicos actores en la escena diplomática: actores supraestatales y subestatales, así como no estatales, cada vez juegan un papel más activo y relevante. Para Janne Nijman (2016), se podría decir que la globalización, urbanización y descentralización actuales remodelan la estructura de poder del Estado y reconfiguran su estructura de autoridad y responsabilidad, empoderando a la ciudad para confrontar asuntos urbanos más activa e independientemente a un nivel global. Como actores subestatales, las ciudades, y especialmente las megaciudades (aquéllas con más de 10 millones de habitantes), participan en la solución de asuntos globales como las migraciones, el terrorismo o el cambio climático. Según Nijman (2016), las ciudades son responsables del 80\% de las emisiones globales de gases de efecto invernadero. Además, más del 50\% de la población mundial vive en ciudades, para 2030 el número habrá aumentado a cerca del $60 \%$ y para 2050 al 75\%. De hecho, para esta autora, las mega e hiperciudades (con más de 20 millones de habitantes) podrían convertirse en las ciudades-Estado del s. XXI.

Según un informe del Centre for European Policy Studies-CEPS (Egenhofer, Alessi, Núñez Ferrer, y Tubiana, 2010), las megaciudades, que han crecido muy rápido en las últimas décadas, se encuentran en países en desarrollo, y su infraestructura no se ha adecuado a ese ritmo de crecimiento. El reto, por lo tanto, sería desarrollar ciudades bajas en carbón y sostenibles a largo plazo, contribuyendo a mitigar el cambio climático. Las ciudades también se enfrentarían al reto de adaptarse al cambio climático, teniendo en cuenta que muchas de ellas se encuentran en la costa y que el aumento de temperatura puede conllevar a problemas de salud y a cambios en la demanda de energía. Como afirma el mencionado informe del CEPS, muchos ya aceptan la visión de que la lucha contra el cambio climático se ganará o perderá en las ciudades. Los Gobiernos locales pueden tener un considerable poder regulatorio y financiero para impulsar inversiones sostenibles de bajo carbono. Además, son proveedores de servicios, consumidores de energía y otros recursos naturales, y compradores de productos y servicios. Bulkeley (2010) añade que la actividad internacional de las ciudades en cambio climático es en gran parte el resultado de la insatisfacción y el sentimiento de fracaso respecto al proceso multilateral puesto en marcha para abordar el problema.

\section{Redes de ciudades y cambio climático}

Las redes de ciudades buscan desarrollar políticas locales eficientes, presionar a Gobiernos nacionales y organizaciones internacionales y crear conciencia en comunidades de todo el mundo (Van der Pluim \& Melissen, 2007). Permiten compartir conocimiento, colaborar para encontrar nuevas soluciones a problemas comunes y aprender de buenas prácticas en otros lugares (Keiner \& Kim, 2007). Para Betsill y Bulkeley (2004) el enfoque en redes transnacionales marca un cambio dentro de la disciplina de relaciones internacionales, de una preocupación por las estructuras jerárquicas a una apreciación de la importancia que tienen formas de organización en red, por ejemplo, en el gobierno medioambiental global. Keiner y Kim (2007) añaden que la creciente influencia de las redes de ciudades demostraría la decreciente importancia de los Estados-nación y el ascenso de una nueva forma de "gobierno glocal". Ya que las megaciudades contribuyen considerablemente a problemas medioambientes como la polución del aire, el calentamiento global, el consumo ineficiente de combustibles fósiles y
Según un informe del CEPS, las megaciudades se encuentran en países en desarrollo, y sus infraestructuras no se han adecuado al ritmo de crecimiento 
energía, la polución del agua, la destrucción de áreas agrícolas o la generación de desperdicio sólido, no es de extrañar que muchas redes de ciudades incluyan entre sus temas y áreas de cooperación el medioambiente, el cambio climático y el desarrollo sostenible.

Jelle Baars (2016) explica cómo las ciudades y otros Gobiernos locales, a través de las redes, han estado presionando a la Convención Marco de Naciones Unidas sobre el Cambio Climático para que reconozca la importancia de la acción climática local. Destaca el Pacto de Ciudad de México, firmado en 2010 en la Cumbre Mundial de Alcaldes que se celebró en Ciudad de México, poco antes de que tuviera lugar la COP16 en Cancún. Mediante el pacto, alcaldes de grandes ciudades firmaron una iniciativa voluntaria por la que se comprometían a reducir las emisiones locales de gases de efecto invernadero de forma medible, reportable y verificable; promover la cooperación entre ciudades; y buscar asociaciones con instituciones multilaterales y Gobiernos nacionales. Otro resultado de la Cumbre Mundial de Alcaldes de 2010 fue la creación del registro climático carbón (Carbon Cities Climate Registry-cCCr), gestionado por ILCEI, mediante el que las ciudades registran sus cifras de emisiones de carbono de manera verificable. En 2012, comenzó a implementarse otro instrumento de medición de emisiones, el Protocolo Global para Inventarios de Emisiones GEI a escala comunitaria (Global Protocol for Community-Scale Greenhouse Gas Emission Inventories-GPC). El GPC es el resultado de un esfuerzo colaborativo entre el World Resources Institute, C40 e ICLEI. Posteriormente se añadieron a la asociación el Grupo Banco Mundial, UN-HABITAT y el Programa de Naciones Unidas para el Medioambiente (PNUMA). El GPC armoniza los procesos de medición e información sobre gases de efecto invernadero para ciudades de todos los tamaños y geografías, y les permite planear y financiar la acción climática. Los participantes del Pacto de México están comprometidos a reportar sus emisiones a esta plataforma. En 2015, Ciudad de México fue premiada por ser "Compliant City" con los cuatro compromisos adquiridos en el Pacto de Alcaldes: firmar el pacto; tener un inventario de emisiones y reportarlo (GPC); tener una meta de mitigación de emisiones (la del PACCM 2014-2020: reducción de 10 millones de toneladas de $\mathrm{CO}_{2}$ eq para 2020) y tener un plan de acción para esa meta (PACCM 2014-2020)ㄹ․

En 2014, la Secretaría General de la ONU organizó la Cumbre de Líderes del Clima, invitando a líderes nacionales, locales, de los negocios y de la sociedad civil a adquirir nuevos compromisos e impulsar nuevas contribuciones contra el cambio climático. En esta cumbre se nombró enviado especial de la ONU para las ciudades y el cambio climático al exalcalde de Nueva York, Michael Bloomberg. Además, en el Acuerdo de París de 2015 se reconoce la importancia de la acción climática local.

Por último, cabe mencionar la creación del Compact of Mayors, una coalición global de alcaldes lanzada en la COP20 de Lima (2014) por el Secretario General de la ONU Ban Ki-Moon y el enviado especial de la ONU para las ciudades y el cambio climático Michael Bloomberg. La coalición fue creada bajo el liderazgo de las redes de ciudades C40, ICLEI y Ciudades y Gobiernos Locales Unidos (United Cities and Local Governments-UCLG), con apoyo del programa de la ONU "Habitat". Dicha coalición reconoce otros compromisos previamente existentes a nivel de ciudad: Acuerdo de Protección del Clima de los Alcaldes Estadounidenses (2005); Pacto de Alcaldes de la Unión Europea-Covenant of Mayors (2008); Campaña Mundial Desarrollando Ciudades Resilientes (2010); Pacto Climático Global de Ciudades-

En el Acuerdo de París de 2015 se reconoce la importancia de la acción climática local 
Pacto de Ciudad de México (2010); y Carta de Adaptación de Durban (2011). El Compact of Mayors establece una plataforma común para recoger el impacto de las acciones colectivas de las ciudades mediante la medición estandarizada de sus emisiones y su riesgo climático, y una información pública y consistente sobre sus esfuerzos (Compact of Mayors, s. f.). En junio de 2016 el Compact of Mayors se fusionó con el Covenant of Mayors², creando el Global Covenant of Mayors for Climate and Energy, que tiene la meta de luchar contra el cambio climático mediante la acción local coordinada.

\section{Iniciativas climáticas de Ciudad de México}

Las iniciativas climáticas emprendidas por Ciudad de México inician el año 2000 con la creación de la Subdirección de Gestión Ambiental y Cambio Climático, dentro de la Secretaría de Medioambiente del Gobierno de la Ciudad de México. Su responsabilidad fue la elaboración del primer inventario de emisiones de gases de efecto invernadero y la primera estrategia de cambio climático. El 30 de agosto de 2007 se dio a conocer el Plan Verde de Ciudad de México, que establecía la ruta del Gobierno para lograr el desarrollo sostenible de la ciudad mediante objetivos y proyectos diseñados para un plazo de 15 años, con una inversión de 1 billón de dólares por año. La Ciudad de México cuenta con la Ley de Mitigación y Adaptación al Cambio Climático y Desarrollo Sustentable para el Distrito Federal (2011), y a nivel nacional se cuenta con la Ley General de Cambio Climático (2012), que convirtió a México en el primer país en desarrollo en contar con una ley sobre cambio climático. Además, en Ciudad de México se han puesto en marcha dos programas específicos de acción climática: el Programa de Acción Climática de la Ciudad de México/PACCM 2008-2012 y el Programa de Acción Climática de la Ciudad de México/PACCM 2014-2020. En el periodo de vigencia del primero se logró una reducción de 6 millones de toneladas de $\mathrm{CO}_{2}$ eq (Leo, Rosa, Cruzado, Reyes, León, et al., 2012). Con el segundo se busca un 30\% de reducción en $\mathrm{CO}_{2}$ eq para 2020: hasta 10 millones de toneladas. En 2017 ya se había logrado mitigar la emisión de 5.3 millones de toneladas de $\mathrm{CO}_{2}$ eq (SEDEMA, 2017). El programa incorpora la perspectiva de género e incluye siete áreas estratégicas con diversas acciones cada una: transición energética urbana y rural; contención de la mancha urbana; mejoramiento ambiental; manejo sostenible de los recursos naturales y conservación de la biodiversidad; construcción de la resiliencia de la ciudad; educación y comunicación; investigación y desarrollo.

Las principales fuentes de emisiones en Ciudad de México son el transporte (51\%), los edificios (32\%) y los residuos (17\%) (C40a, s. f.). El programa ProAire se lanzó en 1995 como instrumento de gestión de la calidad del aire de la ciudad, después de que Naciones Unidas la calificara como la más contaminada del mundo en 1992. ProAire se actualizó en 2001 y 2011 para recalibrar sus metas y hacerlas más estrictas. La versión actual expirará en 2020. El programa recibió el Premio 2013 C40 para la Calidad del Aire en los premios inaugurales de Liderazgo Climático y Ciudad de C40 y Siemens. Ha tenido éxito en la reducción de un $97 \%$ de las emisiones de plomo, el $89 \%$ de $\mathrm{SO}_{2}$, el $79 \%$ de $\mathrm{CO}_{2}$ y el $66 \%$ de PM10, durante un periodo de 25 años. También con objeto de mejorar la calidad del aire, el Programa de Movilidad Integral de Ciudad de México para el periodo 2013-2018 buscó crear una red de transporte
Las principales

fuentes de

emisiones en

Ciudad de México

son el transporte

(51\%), los edificios

(32\%) y los residuos

(17\%)

2 El Covenant of Mayors fue una iniciativa para la cooperación entre autoridades locales y regionales lanzada por la Unión Europea en 2008, con objeto de aumentar el compromiso con el uso de la energía renovable y la eficiencia energética. 
más compacta y eficiente, promoviendo el tránsito en bici y en vehículos de bajas emisiones y reduciendo de esta manera las emisiones de $\mathrm{CO}_{2}$.

Cabe destacar, también, el Programa Voluntario de Certificación de Edificios Sostenibles de Ciudad de México, gestionado por la Secretaría de Medioambiente. Dicho programa se puso en marcha en 2009 como parte del PACCM 2008-2012, y se decidió prolongarlo indefinidamente. Su objetivo es promover la construcción sostenible concediendo una certificación que refleja diferentes niveles de desempeño de sostenibilidad en eficiencia energética, agua, movilidad, residuos sólidos, responsabilidad social y medioambiental, y tejados verdes. La participación voluntaria de propietarios e inquilinos de edificios se incentiva con reducciones de impuestos, de las facturas de luz y agua, acceso a financiación de proyectos, aceleración de procesos de autorización y perspectivas de réditos de renta aumentadas gracias a los suplementos verdes. Desde su creación y hasta 2015 ya se habían certificado 40 edificios, logrando reducciones de consumo de electricidad, agua potable y emisiones de gases de efecto invernadero. En concreto, se logró una reducción de 20.1 millones de Kilovatios de electricidad y de 66.120 toneladas de $\mathrm{CO}_{2}$ eq (Trencher, Takagi, Nishida, y Downy, 2017).

De las dieciséis redes de ciudades en que participa Ciudad de México, ICLEI-Gobiernos Locales por la Sustentabilidad (1990) y el Grupo de Liderazgo Climático-C40 (2005) son las de mayor alcance global y las que más información ofrecen en su página web respecto a iniciativas contra el cambio climático (Coordinación General de Asuntos Internacionales del Gobierno de la Ciudad de México, s. f.). ICLEI dispone de una oficina administrativa en Ciudad de México, mientras que la oficina regional de C40 para Latinoamérica está en Río de Janeiro. En este artículo se va a examinar la colaboración de Ciudad de México con estas dos redes de ciudades, analizando información oficial y realizando entrevistas a trabajadores de la Secretaría de Medioambiente de Ciudad de México, ICLEI y C40.

\section{La colaboración entre Ciudad de méxico e ICLEl- Gobiernos Locales por la Sustentabilidad}

La red de ciudades ICLEI-Gobiernos Locales por la Sustentabilidad (International Council for Local Environmental Initiatives) se creó en 1990, cuando más de 200 Gobiernos locales de 43 países se reunieron en el Congreso Mundial de Gobiernos Locales para un Futuro Sostenible, en la sede de la ONU en Nueva York. La Ciudad de México es socio de ICLEI desde 1999, y su primer intento de crear un Plan de Acción Climática se realizó gracias al intercambio de experiencias y participación en actividades con ICLEI ${ }^{3}$.

El objetivo de ICLEI es representar las preocupaciones medioambientales del Gobierno local internacionalmente y promover iniciativas de desarrollo sostenible dentro del marco de la cooperación descentralizada. A nivel local ICLEI apoya a los Gobiernos locales para que logren la implementación de la Agenda 2030 para el Desarrollo Sostenible. Para ello lleva a cabo campañas de redes, formación y publicaciones.

ICLEI cuenta con diez agendas urbanas, cada una con diversos programas, herramientas y redes. Dentro de la agenda "Ciudades Sustentables" cabe mencionar la participación de Ciu-

3 Entrevista con Paulina Soto, subdirectora de Programas y Proyectos del Secretariado para México, Centroamérica y el Caribe de ICLEI.
El objetivo de ICLEI es representar las preocupaciones medioambientales del Gobiemo local internacionalmente y promover iniciativas de desarrollo sostenible dentro del marco de la cooperación descentralizada 
dad de México en el congreso anual "Ciudades Resilientes" (renombrado en 2012 como Foro Anual Global en Resiliencia Urbana y Adaptación), organizado por ICLEI, el Consejo Mundial de Alcaldes en Cambio Climático y la ciudad de Bonn desde 2010. En la primera reunión los alcaldes firmaron la Declaración de Bonn (2010), que manifiesta su compromiso con la coordinación global de la acción climática local. Estos congresos ofrecen sesiones y eventos en una amplia variedad de temas, como riesgo urbano, logística urbana resiliente, financiación de la ciudad resiliente, agricultura urbana o infraestructura inteligente.

Dentro de la agenda "Ciudad baja en carbón" destaca la herramienta de análisis armonizado de emisiones (Harmonized Emissions Analysis Tool plus-HEAT+), creada por ICLEI para ayudar a los Gobiernos locales a informar sobre emisiones de gases de efecto invernadero, contaminantes comunes del aire y otros componentes orgánicos volátiles. Esta herramienta aporta informes a la plataforma de información internacional para las ciudades llamada Registro Climático carbón. Ciudad de México colabora además con el acelerador de eficiencia de construcción (Building Efficiency Accelerator-BEA), del que es socio ICLEI. BEA es una asociación internacional de múltiples grupos de interés y una red que ayuda a las ciudades y Gobiernos subnacionales a acelerar la adopción de políticas de mejores prácticas y la implementación de proyectos de eficiencia energética en edificios. Dado que los edificios son un contribuidor significativo a las emisiones de gases de efecto invernadero en Ciudad de México, la ciudad fue una de las primeras en unirse a la asociación BEA en 2014, y ha integrado el Proyecto BEA en el PACCM 2014-2020.

En la agenda "Comunidades resilientes" se incluye el Programa de Acciones Transformativas (Transformative Actions Program-TAP), iniciativa que tiene como objetivo catalizar y mejorar los flujos de capital a las ciudades y fortalecer su capacidad para acceder a financiación climática y atraer inversiones para proyectos de adaptación y mitigación. Desde 2015, la Ciudad de México ha participado en tres proyectos TAP en uso de energías renovables, adaptación al cambio climático y eficiencia energética. El primero busca contribuir al ahorro de combustible en la ciudad, proveyendo a los sectores más pobres de fogones de cocina que no emiten gases dañinos. Además, se pretende proveer más energía renovable y aumentar la eficiencia energética de los electrodomésticos. El segundo proyecto aborda una estrategia de resiliencia que busca mantener funciones esenciales durante desastres y aumentar la habilidad de la ciudad de recuperarse rápidamente. La estrategia también considera problemas crónicos dentro de la ciudad, que tienen que ver con la movilidad y el suministro de agua. El tercer proyecto es el Programa Integral para la Eficiencia Energética y el Calentamiento Solar del Agua en 19 hospitales de la ciudad. Respecto a este tercer proyecto, y con el fin de ampliar el radio de acción del mismo, en noviembre de 2017 ICLEI contactó a la Ciudad de México para que participase en el programa FELICITY, logrando de esta manera incrementar el monto y número de edificios. El programa FELICITY es una iniciativa de GIZ, la agencia del Gobierno alemán especializada en cooperación técnica para el desarrollo sostenible que, en colaboración con ICLEI, busca proveer capacidad técnica y asesoría a los municipios para que sus proyectos climáticos sean financiables. Actualmente se está trabajando en una nueva fase para México, donde nuevamente se invitó a la Ciudad de México a presentar proyectos".

Por último, cabe señalar la relevancia que ha adquirido en México el programa Plan de Acción Climática Municipal (PACMUN), aunque Ciudad de México no participa por disponer de su
Como parte de los proyectos TAP, se pretende proveer más energía renovable y aumentar la eficiencia energética de los electrodomésticos 
propio Plan de Acción Climática (PACMM). Desde 2011, el programa PACMUN de ICLEI creó una metodología para desarrollar Planes de Acción Climática Municipal. A través de este proyecto se busca la creación de capacidades de actores municipales para la elaboración de un instrumento que les permita orientar políticas públicas en materia de mitigación y adaptación al cambio climático. Con la elaboración del PACMUN, los municipios crean sus inventarios de gases de efecto invernadero (GEI), y realizan un análisis del grado de vulnerabilidad, para la posterior identificación de acciones de mitigación y adaptación. El PACMUN contó con el respaldo del Instituto Nacional de Ecología y Cambio Climático de la Secretaría de Medioambiente y Recursos Naturales (SEMARNAT) de México durante los años 2011 a 2013, y fue financiado por la embajada británica en México en el periodo 2011-2015. Actualmente ICLEI sigue elaborando Planes de Acción Climática Municipal, ya sea como parte de la afiliación a ICLEI de los Gobiernos locales o como parte de una consultoría, dependiendo de las necesidades de los interesados.

\section{La colaboración entre Ciudad de méxico y C40}

C40 es una red global de ciudades comprometidas en la lucha contra el cambio climático. Se creó en 2005 e incorporó a la Clinton Climate Initiative/CCI en 2006, fusionándose ambas formalmente en 2011. Según su página web, incluye a 96 de las mayores ciudades del planeta, que representan a más de 650 millones de personas y un cuarto de la economía global. La red apoya a estas ciudades para que puedan colaborar y realizar acciones a este respecto (C40b, s. f.). Además, les ayuda en la búsqueda de mayor apoyo y autonomía respecto a los Gobiernos nacionales en la creación de un futuro sostenible (C40c, 2015). C40 tiene numerosos socios aparte de las ciudades, incluyendo fundaciones como Bloomberg Philanthrophies, Children's Investment Fund Foundation y Realdania, y otras redes como ICLEI. Las ciudades C40 se han comprometido a reducir sus emisiones en más de 3 gigatoneladas de $\mathrm{CO}_{2}$ para 2030: el equivalente a quitar 600 millones de coches de la carretera (C40d, 2016).

Una de las primeras colaboraciones destacadas de C40/CCI con Ciudad de México tuvo que ver con la clausura del relleno sanitario Bordo Poniente y con la elaboración de los términos de referencia para la licitación del aprovechamiento del biogás del mismo relleno, en $2011^{5}$. Bordo Poniente era uno de los mayores vertederos del mundo, y Ciudad de México se embarcó en el proyecto de cerrarlo al llegar al límite de su capacidad: 70 millones de toneladas de basura. Con los mencionados proyectos, Ciudad de México planeaba reducir las emisiones de gases de efecto invernadero (hasta 25 millones de toneladas de equivalentes de $\mathrm{CO}_{2}$ en los siguientes 25 años) generando electricidad para los residentes (más de 250 GWh, suficiente para servir a 35.000 hogares). En abril de 2017 se presentó la planta de termovalorización de Bordo Poniente, una iniciativa de Ciudad de México para transformar la basura en energía que comenzará a funcionar en 2019 (Lara, 2017).

Actualmente C40 cuenta con 17 redes organizadas bajo 6 iniciativas, que son: iniciativa de adaptación y agua; iniciativa de energía; iniciativa de finanzas y desarrollo económico; iniciativa de residuos sólidos; iniciativa de transporte; iniciativa de planeación y desarrollo urbano. Dentro de la última iniciativa, la Red de Planeación de Usos del Suelo está liderada por Ciudad de
En abril de 2017 se presentó la planta de termovalorización de Bordo Poniente, una iniciativa de Ciudad de México para transformar la basura en energía que comenzará a funcionar en 2019

5 Entrevista con Rafael Ramos-Villegas, Asesor de C40 para Ciudad de México, 16/04/2018. 
México, a través de la Secretaría de Medioambiente (SEDEMA) y la Secretaría de Desarrollo Urbano y Vivienda (SEDUVI).

C40 cuenta además con una serie de programas diseñados para complementar y profundizar la efectividad de las redes. Estos programas son: Plataforma de Soluciones de Ciudad; Estrategia de Diplomacia de Ciudad; Iniciativa Financiando Ciudades Sostenibles y Unidad para la Financiación de Ciudades. Esta última se lanzó en el foro C40 durante la COP21 de París (2015), y busca contribuir al intercambio de buenas prácticas y de expertos en financiamiento climático que apoyen la preparación de proyectos climáticos de ciudades. La ayuda por parte de C40 consiste en proveer apoyo financiero para recibir asistencia técnica en los proyectos que presentan las ciudades y, en una segunda fase, en proveer asistencia para buscar financiación internacional para la ejecución de los proyectos ${ }^{6}$.

Uno de los dos proyectos piloto de la unidad para la financiación de ciudades, anunciado en septiembre de 2016, consiste en el desarrollo de un corredor de transporte verde con una flota de autobuses limpios eléctricos en Ciudad de México. Se trata de un nuevo "corredor cero emisiones” de autobús que incluirá una flota de autobuses eléctricos y carriles de bicicleta a lo largo del mismo, situado en el Eje 8 Sur, con 22 kilómetros de largo. Servirá para aproximadamente 160.000 viajes diarios, dando conexiones con cinco líneas de metro y una línea de metrobús. Para este proyecto, Ciudad de México recibirá hasta un millón de dólares en ayuda técnica. Cabe destacar que la Ciudad de México fue candidata para la obtención de recursos gracias a su participación constante con C40. El corredor del Eje 8 Sur es uno de los ejemplos de colaboración internacional más concretos y tangibles que ha logrado tener una ciudad ${ }^{7}$.

El proyecto del corredor del Eje 8 Sur sigue la línea del programa C40-CCI de Buses Híbridos y Eléctricos en América Latina, en el que participa Ciudad de México. Lanzado en 2011, su objetivo era reducir la huella de carbono del transporte público en América Latina y desarrollar un mercado de autobuses de bajas emisiones de carbono en la región. Ciudad de México participó introduciendo autobuses híbridos en la línea 4 del sistema de bus de tránsito rápido (Metrobús) $^{8}$. Cabe señalar que el sistema de Metrobús y el sistema de bicicletas compartidas Ecobici comenzaron a implantarse en Ciudad de México desde 2010, y que para 2015 ya eran los sistemas más extensos en América Latina. Los autobuses de tránsito rápido son servicios de autobús de alta velocidad que a menudo operan desde los suburbios de la ciudad hasta el distrito de negocios central, con carriles de autobús separados y con acceso de pasajeros al estilo del metro, incluyendo paradas de autobús protegidas y elevadas por encima de la carretera. Una misma tarjeta electrónica permite acceder a ecobici, metrobús, metro y sistema de tren ligero. La reducción estimada de emisiones gracias a esta integración del transporte público se espera que sea de 13.000 toneladas de $\mathrm{CO}_{2}$ entre 2010 y 2020.

Gracias a su activismo en C40, Ciudad de México ha sido premiada con asesores de ciudad para periodos de dos años (2013-2014; 2015-2016). Los asesores de ciudad son personal dedicado que apoya a ciudades miembro seleccionadas en el desarrollo e implementación de políticas prioritarias, programas y proyectos para reducir las emisiones de gases de efecto invernadero

\section{Ciudad de México participó introduciendo autobuses híbridos en la línea 4 del sistema de bus de tránsito rápido (metrobús)}

6 Entrevista con Patricia Narváez, coordinadora de Asuntos Internacionales de la Secretaría del Medio Ambiente de Ciudad de México (SEDEMA), 20/03/2018.

7 Entrevista con Patricia Narváez, coordinadora de Asuntos Internacionales de la Secretaría del Medio Ambiente de Ciudad de México (SEDEMA), 20/03/2018.

8 Entrevista con Rafael Ramos-Villegas, asesor de C40 para Ciudad de México, 16/04/2018. 
y riesgos climáticos. Desde 2017 y hasta agosto de 2018, Ciudad de México tiene de nuevo un asesor de ciudad, esta vez como parte del proyecto Deadline 2020 de C40, iniciado en 2016. El nuevo asesor, Rafael Ramos-Villegas, elabora un Plan de Acción Climática compatible con el Acuerdo de París para la Ciudad de México, a fin de que en 2050 la ciudad alcance la neutralidad neta de emisiones de carbono ${ }^{9}$.

Otra iniciativa a mencionar es el Foro de Alcaldes Latinoamericanos C40. En marzo de 2015 se celebró la primera edición en Buenos Aires. Se anunció la Declaración de los Alcaldes Latinoamericanos del C40, en la que veinte ciudades de América Latina (incluyendo Ciudad de México) firmaron una declaración regional de compromiso para reducir las emisiones de gases que producen el cambio climático. También se firmó la Declaración de Intenciones de una Ciudad de Autobuses Limpios, que demuestra su compromiso en reducir las emisiones y mejorar la calidad del aire a través de la introducción de autobuses de bajas y cero emisiones en sus flotas. Esta declaración fue también una llamada a los fabricantes, operadores de transporte público, bancos multilaterales de desarrollo y otros organismos de financiación para que apoyen la ambición de lograr un transporte público urbano bajo en carbono.

La sexta Cumbre Bienal de Alcaldes C40 se celebró del 30 de noviembre al 2 de diciembre de 2016, en Ciudad de México. En esta cumbre, Ciudad de México, junto con París, se comprometió a eliminar todos los vehículos diésel para 2025, incentivar vehículos alternativos y promover infraestructura para caminar y andar en bici. Patricia Narváez puntualiza que el compromiso es que el transporte público nuevo adquirido por la ciudad deberá ser híbrido o eléctrico, o en su caso de la menor emisión disponible en el mercado nacional. Se trata de una transición complicada debido a que los manufactureros de este tipo de vehículos son generalmente de fuera del país ${ }^{10}$.

También en 2016, como se mencionó previamente, C40 lanzó el proyecto Deadline 2020, con el que ofrece asesoría técnica a las ciudades C40 para identificar trayectorias de descarbonización con objeto de que cumplan como grupo para evitar el incremento de la temperatura en $1.5^{\circ} \mathrm{C}$, establecido en el Acuerdo de París. La Ciudad de México fue elegida como una de las ocho ciudades piloto para este proyecto (SEDEMA, 2017). Junto con el corredor del Eje 8, la participación en Deadline 2020 podría considerarse uno de los "proyecto estrella” de colaboración de Ciudad de México con C4011.

En 2017 Ciudad de México recibió un premio de la red C40 por su implementación del Segundo Programa de Acción Climática (PACCM 2014-2020). El premio se otorgó en la Cumbre Climática de Alcaldes de Norteamérica, que se llevó a cabo en Chicago. En la cumbre se estableció la "Declaración de Chicago", cuyos objetivos centrales son implementar acciones específicas para asumir el liderazgo en materia climática en América del Norte y alrededor del mundo, adoptar compromisos conjuntos y asumir retos específicos frente al cambio climático. Ciudad de México se comprometió a alinear la meta de mitigación del PACCM 2014-2020 con la establecida en el Acuerdo de París, en colaboración con la iniciativa Deadline 2020. En la cumbre de Chicago, Ciudad de México también se comprometió a reforzar la capacidad de

9 Entrevista con Rafael Ramos-Villegas, Asesor de C40 para Ciudad de México, 16/04/2018.

10 Entrevista con Patricia Narváez, coordinadora de Asuntos Internacionales de la Secretaría del Medio Ambiente de Ciudad de México (SEDEMA), 20/03/2018.

11 Entrevista con Patricia Narváez, coordinadora de Asuntos Internacionales de la Secretaría del Medio Ambiente de Ciudad de México (SEDEMA), 20/03/2018.

\section{La sexta Cumbre} Bienal de Alcaldes C40 de 2016 se comprometió a eliminar todos los vehículos diésel para 2025, incentivar vehículos alternativos y promover infraestructura para caminar y andar en bici 
adaptación de sus habitantes, mediante la implementación de la estrategia de resiliencia de la Ciudad de México.

Por último, Ciudad de México participa en las "prioridades de la presidenta" de C40, que desde 2016 es la alcaldesa de París, Anne Hidalgo. Una de dichas prioridades es la iniciativa "Reinventando Ciudades": una llamada a proyectos urbanos de todo el planeta que presenten caminos innovadores a la neutralidad en carbono y a la regeneración que, si se implementan, podrían transformar sitios infrautilizados en ejemplos de sostenibilidad y resiliencia. Como parte de la iniciativa, las quince ciudades C40 participantes ofrecen sitios de propiedad pública e inmediatamente disponibles para el redesarrollo, llevando a cabo una transferencia de propiedad (venta o alquiler) para permitir a los ganadores implementar su proyecto. La red C40 moviliza a socios privados locales e internacionales para recuperar estas propiedades infrautilizadas, y para invertir en desarrollo verde. Los dos lugares ofrecidos por Ciudad de México, a través de la Secretaría de Desarrollo Urbano y Vivienda (SEDUVI), son el depósito Tetepilco y la subestación Potrero.

Otra de las "prioridades de la presidenta" es la iniciativa Women4Climate, que busca impulsar el liderazgo y la participación de las mujeres en la resolución de la problemática del cambio climático. La primera cumbre de la iniciativa Women4Climate se celebró en Nueva York el 15 de marzo de 2017. En dicha cumbre, alcaldesas de muchas de las mayores ciudades del mundo y mujeres líderes de negocios se comprometieron a usar su creciente poder global para abordar el cambio climático y cumplir con la ambición del Acuerdo de París. La segunda cumbre se celebró en Ciudad de México el 26 de febrero de 2018. La iniciativa incluye un programa de tutoría dedicado a mujeres emprendedoras en iniciativas de cambio climático en las ciudades C40. En Ciudad de México se seleccionaron diez proyectos enfocados a la mitigación o adaptación a los efectos del cambio climático, en el marco de los siete ejes que integran el PACCM 2014-2020 (Gobierno de Ciudad de México, 2018).

\section{Conclusiones}

En el caso de las redes analizadas, C40 e ICLEI, se ha comprobado cómo Ciudad de México ha trabajado de manera conjunta con ambas. Las principales áreas de trabajo conjunto han abordado principalmente la edificación sostenible y el uso sostenible del espacio urbano, el transporte urbano, el ahorro de combustible y la promoción del uso de energías renovables.

Una publicación de la Comisión Europea afirma que el apoyo internacional para que América Latina logre las metas del Acuerdo de París consiste sobre todo en financiamiento internacional, transferencia de tecnología y fortalecimiento de capacidades (Karremans, Brugger, Castillo, Argüello, y Dascal, 2017). En efecto, en esta investigación se ha comprobado cómo en el caso de Ciudad de México, la colaboración con ICLEI y C40 enfatiza la ayuda técnica y los programas de preparación para la financiación externa de proyectos climáticos, como ocurre con el programa Unidad para la Financiación de Ciudades de C40 y con los programas TAP y FELICITY de ICLEI.

Este artículo permite concluir que Ciudad de México ha adoptado, desde hace más de una década, una actitud proactiva como actor local en la lucha contra el cambio climático. Además de participar en los diferentes foros, pactos y cumbres que abordan el papel a ejercer por las ciudades en la resolución de este problema, ha emprendido iniciativas propias y ha colaborado estrechamente con redes de ciudades. Las iniciativas y colaboración de Ciudad de México

\section{La Comisión} Europea afima que el apoyo internacional para que América Latina logre las metas del Acuerdo de París consiste sobre todo en financiamiento internacional, transferencia de tecnología y fortalecimiento de capacidades 
con redes de ciudades son una muestra de acción climática local y descentralización de las relaciones internacionales. En definitiva, del empoderamiento de las ciudades para enfrentar problemas globales a nivel local, de forma activa y relativamente independiente.

\section{Bibliografía}

Baars, J. (2016). Motors of Change? A Study on Cities and Climate Change. (Master Thesis. MAIS International Studies, Leiden University). Recuperado de https://openaccess.leidenuniv.nl/handle/1887/42087

Betsill, M., \& Bulkeley, H. (2004). Transnational Networks and Global Environmental Governance: The Cities for Climate Protection Program. International Studies Quarterly, 48(2), 471-93. DOI: https://doi.org/10.1111/j.0020-8833.2004.00310.x

Bulkeley, H. (2010). Cities and the Governing of Climate Change. Annual Review of Environment and Resources, 35, 229-53.

C40a (sin fecha). Ciudad de México. Recuperado de http://www.c40.org/cities/ciudad-demexico\#city-header

C40b (sin fecha). About C40. Recuperado de http://www.c40.org/about

C40c (diciembre 2015). Why Cities? C40 Factsheet. Recuperado de https://c40-productionimages.s3.amazonaws.com/fact_sheets/images/5_Why_Cities_Dec_2015.original. pdf? 1448476459

C40d (octubre 2016). C40 Cities, 10 years of results. Recuperado de https://c40-productionimages.s3.amazonaws.com/fact_sheets/images/11_C40_on_its_10-year_anniversary_ reOct2016.original.pdf? 1475504972

Compact of Mayors (sin fecha). Recuperado de https://www.compactofmayors.org/history/

Coordinación General de Asuntos Internacionales del Gobierno de la Ciudad de México (sin fecha). Recuperado de http://www.internacionales.cdmx.gob.mx/index.php/es/cdmxinternacional/redes-de-ciudades

Egenhofer, C., Alessi, M., Núñez Ferrer, J. y Tubiana, L. (2010). Greening EU Cities: The Emerging EU Strategy to Address Climate Change. Bruselas: Centre for European Policy Studies. Recuperado de https://www.ceps.eu/publications/greening-eu-citiesemerging-eu-strategy-address-climate-change

Gobierno de Ciudad de México (2018). Anuncian a las 10 ganadoras del programa Tutoría CDMX "Mujeres por el Clima”. Recuperado de http:/www.cdmx.gob.mx/comunicacion/ nota/anuncian-las-10-ganadoras-del-programa-tutoria-cdmx-mujeres-por-el-clima

Keiner, M., \& Kim, A. (2007). Transnational City Networks for Sustainability. European Planning Studies, 15(10), 1369-1395.

Lara, R. (2017). La planta que dará energía a la CDMX con basura operará en 2019, Obrasweb, 05/09/2017. Recuperado de http://obrasweb.mx/construccion/2017/09/05/la-plantaque-dara-energia-a-la-cdmx-con-basura-operara-en-2019

Leo, J., Rosa, A. de la, Cruzado, A., Reyes, A., González, C., León, D. y otros (2012). Evaluación del Programa de Acción Climática de la Ciudad de México 2008-2012. México D.F.: 
Centro Mario Molina para Estudios Estratégicos sobre Energía y Medio Ambiente A. C. Recuperado de http://centromariomolina.org/wp-content/uploads/2012/12/ResumenEjecutivo-PACCM-2008-2012-CMM_fin.pdf

Nijman, J. (2016). Renaissance of the City as a Global Actor. The Role of Foreign Policy and International Law Practices in the Construction of Cities as Global Actors (Research Paper Series). La Haya: Asser Institute, Center for International \& European Law. Recuperado de https://papers.ssrn.com/sol3/papers.cfm?abstract_id=2737805

Secretaría del Medioambiente de la Cuidad de México/SEDEMA (S. F.). ¿YY la CDMX qué ha hecho para reducir los efectos del cambio climático? Recuperado de http://www.data. sedema.cdmx.gob.mx/cambioclimaticocdmx/cdmx.html

Secretaría del Medioambiente de la Cuidad de México/SEDEMA (2017). Quinto Informe de Gobierno. Recuperado de http://www.sedema.cdmx.gob.mx/storage/app/uploads/ public/5al/db0/045/5aldb00453394630961121.pdf

Trencher, G., Takagi, T., Nishida, Y., y Downy, F. (2017). Urban Efficiency II. Seven Innovative City Programmes for Existing Building Energy Efficiency. Tokyo Metropolitan Government Bureau of Environment, C40 Cities Climate Leadership Group, CSR Design Green Investment Advisory, Co., Ltd. London.

Van der Pluim, R., \& Melissen, J. (April 2007). City Diplomacy: The Expanding Role of Cities in International Politics. Netherlands Institute of International Relations Clingendael. Recuperado de https://www.uclg.org/sites/default/files/20070400_cdsp_paper_pluijm.pdf 